\title{
Medication used to control symptoms of chronic urticaria in children
}

\author{
Kantima Kanchanapoomi, Punchama Pacharn, Nualanong Visitsunthorn, Orathai Jirapongsananuruk
}

\begin{abstract}
Background: A number of guidelines for management of CU were established based on evidences in adults. In children, the response to CU treatment was not widely studied.

Objective: To investigate the medications used to control symptoms of CU in children and to identify factors associated with time to control CU.

Methods: Medical records of children with controlled CU visiting Allergy clinic, Siriraj hospital were examined. Controlled CU was defined as no urticarial lesion while the patients used the same daily medications over 8-12 weeks. Demographic data, clinical progression of $\mathrm{CU}$ and medications used in each visit were recorded. The steps of CU management were categorized into groups according to the Joint Task Force Practice Parameter (JTFPP) in CU 2014 guideline.

Results: One hundred children (48 males) with 'controlled CU' were recruited. The median age at first visit was 8 (5.4010.60) years. Thirty-two percent of the patients had associated angioedema. The median time to control CU was 9 (6.9011.10) months. Forty-four percent of the patients control CU with standard dose of second generation antihistamine (step 1) and the rest of the patients used the medications in step 2 to control CU. None of the patients needed systemic corticosteroid or immunomodulatory agent. The steps of treatment, angioedema and associated conditions related to CU did not affect time to control.
\end{abstract}

Conclusion: Only up to a half of pediatric patients with CU had a favorable response to standard dose of second generation antihistamine. The rest required step 2 treatment of JTFPP to control their symptoms.

Key words: angioedema, antihistamine, children, chronic urticaria, chronic spontaneous urticaria, urticaria

\section{From:}

Division of Allergy and Immunology, Department of Pediatrics, Faculty of Medicine, Siriraj Hospital, Mahidol University, Bangkok, Thailand

\section{Introduction}

Chronic urticaria (CU) is defined as urticaria that presents continuously or almost every day for at least 6 weeks. ${ }^{1,2}$ The prevalence of $\mathrm{CU}$ was $0.5-5 \%$ in general population and the incidence of CU was estimated to be $1.4 \%$ per year. ${ }^{2,3}$ In children, the prevalence of CU in the UK was $0.1 \%-0.3 \%$. In a Thai study, $13 \%$ of children with urticaria were diagnosed as having CU. ${ }^{4}$

CU subtypes include chronic spontaneous urticaria (CSU) and inducible urticaria. ${ }^{1}$ Disorders identified as possible causes of CSU included food or drug allergies, autoimmune diseases, chronic infections, and parasitic infestation. ${ }^{5}$ More than $30 \%$

\section{Corresponding author:}

Orathai Jirapongsananuruk

Division of Allergy and Immunology, Department of Pediatrics,

Faculty of Medicine Siriraj Hospital, Mahidol University,

2 Wanglang Road, Bangkoknoi, Bangkok, Thailand, 10700

E-mail: jirapongo@yahoo.com

of patients with CSU had autoantibodies to the a-subunit of high-affinity IgE receptor (FceRIa) and/or anti-IgE antibodies, and were classified as having chronic autoimmune urticaria (CAU).$^{6-13}$ Sixty percent of CSU remained idiopathic. ${ }^{5}$

The detailed history and physical examination are most important diagnostic tools for CU diagnosis. The yield of laboratory tests to identify the etiology of CU was low and the causal relationship was not clear. ${ }^{14,15} \mathrm{CU}$ in children was different from that in adults. ${ }^{16,17}$ The goal of CU treatment is to avoid the triggering factors, control urticarial symptoms, and minimize treatment related side effects. 
Recently, multi-national guidelines for the treatment of CU have been established e.g. the European Academy of Allergy and Clinical Immunology, Global Allergy and Asthma European Network, European Dermatology Forum \& World Allergy Organization (EAACI/GA²LN/EDF/WAO) for management of urticaria 2013, Joint Task Force Practice Parameter (JTFPP) in CU 2014 (USA) and Clinical practice guideline for the diagnosis and management of urticaria of Thailand 2014. ${ }^{1,2,18}$ All guidelines emphasized a stepwise approach for urticarial symptom control with some minor differences. The first line treatment in all guideline is second generation antihistamines. The second-line treatment in EAACI/GA²LEN/ $\mathrm{EDF} / \mathrm{WAO}$ guideline is updosing of second generation antihistamine to fourfold. In Thai guideline, the second step is the same as EAACI/GA ${ }^{2} \mathrm{LEN} / \mathrm{EDF} / \mathrm{WAO}$ guideline but has the option to add another second generation antihistamine. In the JTFPP guideline, the second step provides many regimens which include one or more of increasing dose of the second generation antihistamine, adding $\mathrm{H} 2$-antagonist, montelukast or the first generation antihistamine at bedtime. The third line in EAACI/GA ${ }^{2} \mathrm{LEN} / \mathrm{EDF} / \mathrm{WAO}$ guideline recommends adding montelukast, cyclosporine A or omalizumab. Thai guideline is the same as EAACI/GA ${ }^{2} \mathrm{LEN} / \mathrm{EDF} / \mathrm{WAO}$ guideline but has the option to add $\mathrm{H} 2$ antagonist in the third step. There are the third and forth steps of treatment in JTFPP guideline. The dose advancement of sedating first generation antihistamine as tolerated is the third step and adding alternative agents such as omalizumab, cyclosporine $\mathrm{A}$, or other immunomodulatory agents is the forth step of treatment.

These guidelines, however, were extrapolated from the studies in adults. The response to CU management in children was not widely investigated. The objective of this study was to investigate the medications used to control the symptoms of $\mathrm{CU}$ in Thai children and to identify factors associated with time to control CU in these patients.

\section{Methods}

\section{Study design and subjects}

This study was approved by the Institutional Review Boards, Faculty of Medicine Siriraj Hospital, Mahidol University. NO. $316 / 2559$, COA No. Si 396/2016. The medical records of children aged 4-18 years with controlled CU were longitudinally reviewed. These patients were followed by pediatric allergists from June 2001 to May 2016, in the Pediatric allergy clinic, Siriraj Hospital Mahidol University, Thailand.

\section{Data collection}

All data of the patients were collected in the case record form, including demographic data (age, sex, angioedema, associated cause, onset of $\mathrm{CU}$ ), the clinical progression of $\mathrm{CU}$ and medication used in each visit.

\section{Steps of treatment}

The pediatric allergists used medications to control CU according to the CU guidelines suggested by the EAACI/GALEN/EDF/WAO, JTFPP, or Thai guideline. The medications used in each visit were recorded. Due to the broad variation in treatment regimens, the final medications to control CU were

categorized into group according to the JTFPP guideline as in Figure 1. The usual follow up time was 8 to 12 weeks. The pediatric allergists stepped up treatment after ensuring that the patients had good compliance without symptom.

Step 1

- Standard dose of second generation antihistamine

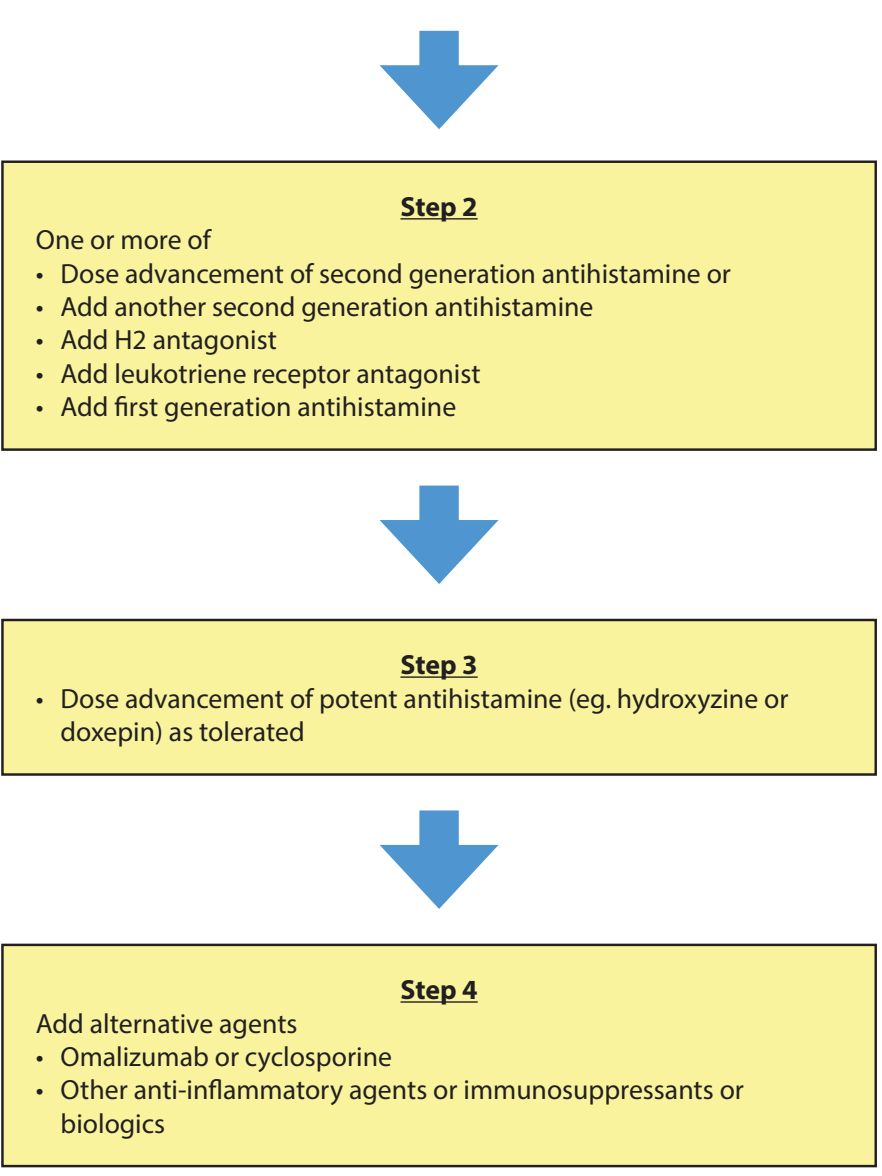

Figure 1. The steps of chronic urticaria treatment in this study

\section{The definition of 'controlled chronic urticaria' and time to con- trol}

The definition of 'controlled CU' was no urticarial lesion while the patients were on the same daily medications over subsequent outpatient visits which was usually spaced 8 to 12 weeks apart. ${ }^{19}$ 'Time to control CU' was the duration from the date of diagnosis to the date that patients had 'controlled CU'.

\section{Statistical analysis}

Demographic and characteristic data were analyzed by descriptive statistics using median (range). The Kaplan-Meier survival curve was used to analyze the probability of 'controlled CU' and the relation between time to control CU and angioedema, associated causes, and medication regimens. The statistical significance was determined by a log rank test. A $\mathrm{p}$ value less than 0.05 was considered significant. Statistical analyses were performed using SPSS version 18. 


\section{Results}

Demographic data and characteristics of the patients

The medical records of 100 children (48 males) with 'controlled CU', were reviewed. The demographic characteristics of these patients were shown in Table $\mathbf{1}$. The median age at the first visit was 8 years and associated angioedema was found in $32 \%$ of the patients. The median time from onset of CU to the diagnosis was 5 months.

Information obtained from the medical records revealed that most patients did not have identified cause (80\%). The autologous serum skin test (ASST) was done in 19 children.

Table 1. Demographic characteristics of pediatric patients with chronic urticaria $(n=100)$

\begin{tabular}{|c|c|}
\hline Characteristics & Value \\
\hline Median age (yr) (range) & $8(4-18)$ \\
\hline Sex: Male (n, \%) & $48(48 \%)$ \\
\hline Angioedema (n, \%) & $32(32 \%)$ \\
\hline Median (range) time from onset to diagnosis (months) & $5(2,120)$ \\
\hline \multicolumn{2}{|l|}{ Condition related to urticaria $(n, \%)$} \\
\hline Un-identify & $80(80 \%)$ \\
\hline Autoimmune & $12(12 \%)$ \\
\hline Autologous serum skin test positive & $10(10 \%)$ \\
\hline Autoimmune thyroiditis & $2(2 \%)$ \\
\hline Food & $4(4 \%)$ \\
\hline Parasitic infestation & $1(1 \%)$ \\
\hline Physical factor & $3(3 \%)$ \\
\hline
\end{tabular}

Ten patients had positive ASST and were diagnosed as having chronic autoimmune urticaria (CAU). Antibodies to thyroid were investigated in 45 children ( 1 had positive antithyroglobulin (anti-Tg) plus antithyroid peroxidase (anti-TPO) and 1 had positive anti-TPO. Seven patients had suspected history of food allergy and skin prick test positive to food. Oral food challenges were done in 5 patients and 4 patients had positive challenges ( 3 to shrimp, 1 to egg white). These 4 patients had improved urticaria after diet elimination. One patient had positive gnathostoma antibody. He was treated with anti-parasite medication and urticaria was gradually improved. Physical factors associated with urticaria were found in 3 patients ( 2 with delayed pressure and 1 with symptomatic dermatographism).

\section{Medications used to control chronic urticaria}

The medications used to control CU were shown in Table 2. Forty-four patients achieved control of CU by using standard dose of the second generation antihistamine (step 1). The rest of the patients achieved symptom control by using the medications in step 2. The most common regimen used in step 2 was increased doses of second generation antihistamine (19\%), followed by standard dose of second generation antihistamine plus $\mathrm{H} 2$ blocker (10\%) and increased doses of second generation antihistamine plus $\mathrm{H} 2$ blocker (10\%). None of the patient needed systemic corticosteroid or medications in step 3 or 4 .

The median time to control CU in all patients was $9(95 \%$ confidential interval (CI) 6.90-11.10) months. The median time to control CU in step 1 and 2 regimens were 8 (95\% CI 5.40-10.60) months and 10 (95\% CI 8.67-11.33) months, respectively, $p=0.13$ ). In the step 2 , the median time to control $\mathrm{CU}$ using increased doses of second generation antihistamine was 11 (95\% CI 9.35-12.65) months, standard dose of second

Table 2. Number of patients with 'controlled chronic urticaria' and median time to control chronic urticaria in each medication regimen

\begin{tabular}{|c|c|c|}
\hline Medication to control chronic urticaria & $\begin{array}{l}\text { Number of patient with } \\
\text { controlled CU }(\%)(n=100)\end{array}$ & $\begin{array}{l}\text { Median time to control CU } \\
(95 \% \mathrm{CI}) \text { (months) }\end{array}$ \\
\hline Step 1: & $44(44 \%)$ & $8(5.40,10.60)$ \\
\hline \multicolumn{3}{|l|}{ Standard dose of second generation $\mathrm{AH}$} \\
\hline Step 2: & $56(56 \%)$ & $10(8.67,11.33)$ \\
\hline - Increased doses of second generation $\mathrm{AH}$ & $19(19 \%)$ & \\
\hline - Standard dose of second generation $\mathrm{AH}+\mathrm{H} 2$ blocker & $10(10 \%)$ & \\
\hline - Increased doses of second generation $\mathrm{AH}+\mathrm{H} 2$ blocker & $10(10 \%)$ & \\
\hline - Standard dose of second generation $\mathrm{AH}+$ first generation $\mathrm{AH}$ & $7(7 \%)$ & \\
\hline - Increased doses of second generation $\mathrm{AH}+\mathrm{H} 2$ blocker + LTRA & $3(3 \%)$ & \\
\hline - Standard dose of second generation $\mathrm{AH}+\mathrm{H} 2$ blocker + first generation $\mathrm{AH}$ & $2(2 \%)$ & \\
\hline - Increased doses of second generation $\mathrm{AH}+$ first generation $\mathrm{AH}$ & $2(2 \%)$ & \\
\hline - Standard dose of second generation $\mathrm{AH}+\mathrm{H} 2$ blocker + LTRA & $1(1 \%)$ & \\
\hline - Standard dose of second generation $\mathrm{AH}+\mathrm{H} 2$ blocker + first generation $\mathrm{AH}+\mathrm{LTRA}$ & $1(1 \%)$ & \\
\hline - Increased doses of second generation $\mathrm{AH}+\mathrm{H} 2$ blocker + first generation $\mathrm{AH}$ & $1(1 \%)$ & \\
\hline
\end{tabular}

AH, antihistamine; LTRA, leukotriene receptor antagonist 
generation antihistamine plus $\mathrm{H} 2$ blocker was 10 (95\% CI 3.93-16.07) months, standard dose second generation antihistamine plus first generation antihistamine was 7 (95\% CI 1.8712.13) months, increased doses of second generation antihistamine plus $\mathrm{H} 2$ blocker was 8 (95\% CI 6.48-9.52) months and increased doses of second generation antihistamine plus $\mathrm{H} 2$ blocker plus leukotriene receptor antagonist (LTRA) was 14 (95\% CI 0.00-31.60) months. Other regimens in step 2 were not evaluated due to the limited number of patients.

Two CU patients were diagnosed as having autoimmune thyroiditis. Two of them had abnormal thyroid function tests (high free thyroxine and low thyroid stimulating hormone) and were treated with thyroxine. One patient had normal thyroid hormone and did not receive any thyroid medication.

\section{Factors affected time to control chronic urticaria}

The relations between angioedema or associated causes and time to control CU were investigated. The Kaplan-Meier survival curve showed that the angioedema group could control CU in 10 (95\% CI 8.15-11.85) months and the non-angioedema group in 9 (95\% CI 6.80-11.20) months $(p=0.84)$ (Figure 2).

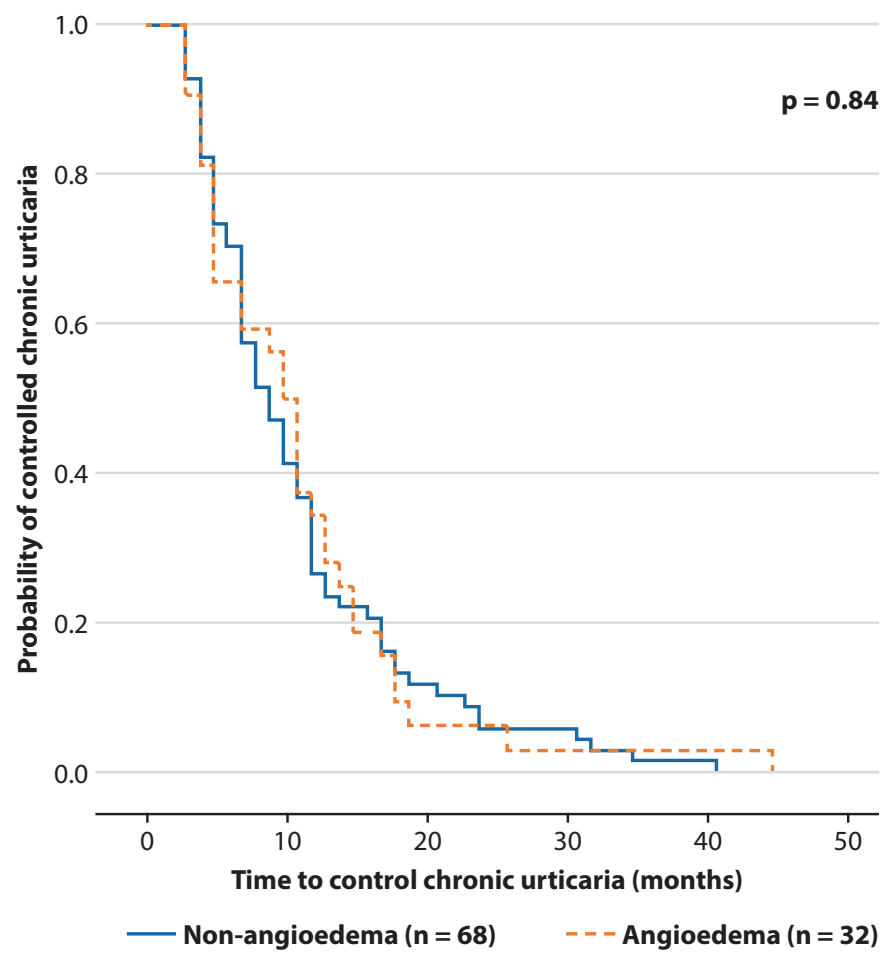

Figure 2. The probability of the 'controlled chronic urticaria' in angioedema versus non - angioedema groups

The median time to control CU was 10 (95\% CI 7.98-12.02) months in the unidentified cause, 5 months in autoimmune thyroiditis, 5 (95\% CI 4.15-5.85) months in food induced CU, 17 months in physical urticaria, and 12 months in parasitic infestation $(p=0.26)$. The $95 \% \mathrm{CI}$ of autoimmune thyroiditis, parasitic infestation and physical urticaria groups could not be calculated due to the limited number of patients. The ASST negative group could control CU in 10 (95\% CI 8.26-11.74) months and the ASST positive group (CAU) in 7 (95\% CI 3.9610.04) months $(p=0.25)$.

\section{Discussion}

$\mathrm{CU}$ in children was different from that in adults. ${ }^{16,17}$ There was no sex difference in children, while CU was found to be twice as frequent in female patients. ${ }^{15,16}$ Moreover, less autoimmune thyroiditis and connective tissue disease were associated with CU in children compared to adult. ${ }^{20-24}$

A number of studies investigated the response of CU treatment in adults. A retrospective study of adult patients with $\mathrm{CU}$ by Amin et al revealed that $63 \%$ of the patients achieved complete urticarial control. ${ }^{25}$ Using the JTFPP recommendation, $56 \%$ of patients received step 1-3 regimens to completely control CU. The most effective regimen was a combination of second generation antihistamine plus LTRA (22\%), followed by a combination of second generation antihistamine plus H2-blocker (13.8\%). ${ }^{25}$ The step 4 treatment was used in $44 \%$ of the patients and the most effective regimen was either cyclosporine $(33 \%)$ or omalizumab (33\%) in addition to the existing medication regimen. ${ }^{25}$

A cross-sectional study in a dermatology clinic in a tertiary hospital in Spain, demonstrated that the standard dose of second generation antihistamine was sufficient to control CSU in $18 \%$ of the patients. ${ }^{26}$ The additional control of $21 \%$, $16 \%$ and $19 \%$ were found when the dose adjustment were increased to 2, 3 and 4 times of the standard dose, respectively. ${ }^{26}$ Twenty-five percent of the patients failed to respond to second generation antihistamines and required additional treatment with omalizumab or cyclosporine to control CU. ${ }^{26}$

A recent multicenter, triple-blinded, prospective, randomized study in 2 Colombian cities recruited $150 \mathrm{CSU}$ patients older than 12 years and treated according to the EAACI/GALEN/EDF/WAO guideline. ${ }^{27}$ The first line treatment with standard dose of second generation antihistamine induced clinical response in $59 \%$ of the patients. An addition of $17 \%$ achieved clinical response with the second-line treatment of higher dose second generation antihistamine. Other $15 \%$ of the patients reached a good clinical control with the use of omalizumab (8\%) and cyclosporine (7\%). Of interest, the control rate with first line treatment was superior at 1 month than at 2 weeks $(p$ $<0.0001){ }^{27}$

Taken together, these adult studies indicated that a majority of CU could achieve symptom control with the dose adjustment of second generation antihistamine. However, 15-44\% of adult patients needed immunomodulatory agents to control $\mathrm{CU}^{25-27}$ In children, a relatively few publications explored the treatment response of CU. In a prospective study by Lee et al in Singapore, 98 children with CSU were treated in a stepwise weight-adjusted management with second generation antihistamine ${ }^{19}$ Half of the children could control CU with standard dose of second generation antihistamine. The addition of $36 \%$, $6 \%$ and $5 \%$ of the children could control CU with 2,3 and 4 times of the standard dose. Of note, none of the patients needed cyclosporine or omalizumab to control CU. ${ }^{19}$ Our study also supported the findings by Lee et al that almost half of CU children could achieve urticarial control by using standard dose of second generation antihistamine. The rest of patients could achieve urticarial control by using the step 2 of JTFPP recommendation. None of the patients needed systemic steroid, cyclosporine or omalizumab, suggesting that $\mathrm{CU}$ in children had a favorable outcome and could be controlled without 
using immunomodulatory agents. The medication steps used to control CU in adults are more than steps in children. It might be due to the higher incidence of collagen vascular disease associated with CU in adults than in children. ${ }^{15,24}$

An adult study by Amin et al, revealed that the average time to control was $1.4-2.7$ years. ${ }^{25}$ A pediatric study by Lee et al showed that the median time to control CSU by using a standard dose of second generation antihistamine, 2, 3 and 4 times of the standard dose, were $2,8,9.5$, and 14.5 weeks, respectively. ${ }^{19}$ The median time to control CU in our patients was 9 months which was shorter than that in adult study but was longer than a pediatric study. It might be due to the longer duration of follow-up period (8-12 weeks) in our study compared to 6-12 weeks in the study by Lee et al. ${ }^{19}$

Factors associated with a poorer CU control were identified in a number of studies. These factors included chronic inducible urticaria, angioedema, anti-thyroid antibodies and positive ASST. ${ }^{25,26,28,29,30}$ Amin et al reported that the odd ratio of poor controlled CU in physical urticaria was 1.5 compared to those without physical urticaria. ${ }^{25}$ The study by MarinCabanas et al indicated that patients with angioedema were associated with an up to 6-fold greater risk of not response to second generation antihistamine than patients who did not have angioedema. ${ }^{26}$ Toubi et al showed that the longer duration of $\mathrm{CU}$ was associated with the presence of anti-thyroid antibodies and positive ASST. ${ }^{29}$ However, conflicting results of the association between CU duration and angioedema, anti -thyroid antibodies and positive ASST were shown in a systematic review by Rabelo-Filardi et al. ${ }^{30}$ Our study investigated factors associated with time to control CU. The treatment steps, presence of angioedema or associated causes, were not significantly affected the time to control CU.

The limitation of this study was that the retrospective chart review might inadequately provided information which was needed. The pediatric allergists did not investigate all patients. The investigation was done in some patients based on their suggestive history. The different medication regimens, variation in stepping up the medications, follow up period and judgement on investigation depended on different pediatric allergists in accordance with different guidelines. However, we believed our data reflected the real life managemnet of CU in children. Since the aim of this study was to investigate the medications used to control the symptoms of $\mathrm{CU}$ in Thai children, the total number of patients with CU and the proportion of CU remission during the study period were not recorded.

\section{Conclusion}

Our study demonstrated that almost half of pediatric patients with $\mathrm{CU}$ had a favorable response to standard dose of second generation antihistamine. The rest of them could control the symptoms by using the step 2 treatment of JTFPP recommendation. None of the patients required prednisolone, immunosuppressive, or biologic agents.

\section{Acknowledgements}

We would like to thank Dr. Saowalak Hunnangkul from Clinical Epidemiology Unit, Office for Research and Development, and Miss. Kanokwan Sommai from Pediatrics Department, Faculty of Medicine, Siriraj Hospital, Mahidol University for statistical consultation.

\section{References}

1. Zuberbier T, Aberer W, Asero R, Bindslev-Jensen C, Brzoza Z, Canonica GW, et al. The EAACI/GA(2) LEN/EDF/WAO Guideline for the definition, classification, diagnosis, and management of urticaria: the 2013 revision and update. Allergy. 2014;69:868-87.

2. Bernstein JA, Lang DM, Khan DA, Craig T, Dreyfus D, Hsieh F, et al. The diagnosis and management of acute and chronic urticaria: 2014 update. J Allergy Clin Immunol. 2014;133:1270-7.

3. Quirt J, Lima H, Wasweman S. Urticaria a multidisciplinary disease. where are we now? Curr Derm Rep. 2015;4:8-14.

4. Tuchinda M, Srimaruta N, Habanananda S, Vareenil J, Assatherawatts A. Urticaria in Thai children. Asian Pac J Allergy Immunol. 1986;4:41-5.

5. Greaves MW, Tan KT. Chronic urticaria: recent advances. Clin Rev Allergy Immunol. 2007;33:134-43.

6. Fiebiger E, Maurer D, Holub H, Reininger B, Hartmann G, Woisetschlager $\mathrm{M}$, et al. Serum IgG autoantibodies directed against the alpha chain of Fc epsilon RI: a selective marker and pathogenetic factor for a distinct subset of chronic urticaria patients? J Clin Invest. 1995;96:2606-12.

7. Niimi N, Francis DM, Kermani F, O’Donnell BF, Hide M, Kobza-Black A, et al. Dermal mast cell activation by autoantibodies against the high affinity IgE receptor in chronic urticaria. J Invest Dermatol. 1996;106:1001-6.

8. Ferrer M, Kinet JP, Kaplan AP. Comparative studies of functional and binding assays for IgG anti-Fc(epsilon)RIalpha (alpha-subunit) in chronic urticaria. J Allergy Clin Immunol. 1998;101:672-6.

9. Fiebiger E, Hammerschmid F, Stingl G, Maurer D. Anti-FcepsilonRIalpha autoantibodies in autoimmune-mediated disorders. Identification of a structure-function relationship. J Clin Invest. 1998;101:243-51.

10. Sabroe RA, Grattan CE, Francis DM, Barr RM, Kobza Black A, Greaves MW. The autologous serum skin test: a screening test for autoantibodies in chronic idiopathic urticaria. Br J Dermatol. 1999;140:446-52.

11. Asero R, Tedeschi A, Lorini M, Salimbeni R, Zanoletti T, Miadonna A. Chronic urticaria: novel clinical and serological aspects. Clin Exp Allergy. 2001;31:1105-10.

12. Boguniewicz $M$. The autoimmune nature of chronic urticaria. Allergy Asthma Proc. 2008;29:433-8.

13. Greaves MW. Chronic urticaria in childhood. Allergy. 2000;55:309-20.

14. Kozel MM, Bossuyt PM, Mekkes JR, Bos JD. Laboratory tests and identified diagnoses in patients with physical and chronic urticaria and angioedema: A systematic review. J Am Acad Dermatol. 2003;48:409-16.

15. Jirapongsananuruk O, Pongpreuksa S, Sangacharoenkit P, Visitsunthorn $\mathrm{N}$, Vichyanond P. Identification of the etiologies of chronic urticaria in children: a prospective study of 94 patients. Pediatr Allergy Immunol. 2010;21:508-14.

16. Choi SH, Baek HS. Approaches to the diagnosis and management of chronic urticaria in children. Korean J Pediatr. 2015;58:159-64.

17. Church MK, Weller K, Stock P, Maurer M. Chronic spontaneous urticaria in children: itching for insight. Pediatr Allergy Immunol. 2011;22:1-8.

18. Kulthanan K, Tuchinda P, Chularojanamontri L, Chanyachailert P, Korkij W, Chunharas A, et al. Clinical practice guideline for diagnosis and management of urticaria. Asian Pac J Allergy Immunol. 2016;34:190-200.

19. Lee XH, Ong LX, Cheong JY, Sultana R, Rao R, Lim HH, et al. A stepwise approach in the management of chronic spontaneous urticaria in children. Asia Pac Allergy. 2016;6:16-28.

20. Sharma M, Bennett C, Carter B, Cohen SN. H1-antihistamines for chronic spontaneous urticaria: an abridged Cochrane Systematic Review. J Am Acad Dermatol. 2015;73:710-6.e4.

21. Church MK. Safety and efficacy of bilastine: a new H(1)-antihistamine for the treatment of allergic rhinoconjunctivitis and urticaria. Expert Opin Drug Saf. 2011;10:779-93.

22. Sahiner UM, Civelek E, Tuncer A, Yavuz ST, Karabulut E, Sackesen C, et al. Chronic urticaria: etiology and natural course in children. Int Arch Allergy Immunol. 2011;156:224-30. 
23. Liutu M, Kalimo K, Uksila J, Kalimo H. Etiologic aspects of chronic urticaria. Int J Dermatol. 1998;37:515-9.

24. Kulthanan K, Jiamton S, Thumpimukvatana N, Pinkaew S. Chronic idiopathic urticaria: prevalence and clinical course. J Dermatol. 2007;34: 294-301.

25. Amin P, Levin L, Holmes SJ, Picard J, Bernstein JA. Investigation of patient-specific characteristics associated with treatment outcomes for chronic urticaria. J Allergy Clin Immunol Pract. 2015;3:400-7.

26. Marin-Cabanas I, Berbegal-de Gracia L, de Leon-Marrero F, Hispan P, Silvestre JF. Management of Chronic Spontaneous Urticaria in Routine Clinical Practice Following the EAACI/GA(2)LEN/EDF/WAO Guidelines. Actas Dermosifiliogr. 2017;108:346-53.
27. Sanchez J, Zakzuk J, Cardona R. Evaluation of a Guidelines-Based Approach to the Treatment of Chronic Spontaneous Urticaria. J Allergy Clin Immunol Pract. 2018;6:177-82 e1.

28. Kocaturk E, Can PK, Akbas PE, Copur M, Degirmentepe EN, Kiziltac K, et al. Management of chronic inducible urticaria according to the guidelines: A prospective controlled study. J Dermatol Sci. 2017;87:60-9.

29. Toubi E, Kessel A, Avshovich N, Bamberger E, Sabo E, Nusem D, et al. Clinical and laboratory parameters in predicting chronic urticaria duration: a prospective study of 139 patients. Allergy. 2004;59:869-73.

30. Rabelo-Filardi R, Daltro-Oliveira R, Campos RA. Parameters associated with chronic spontaneous urticaria duration and severity: a systematic review. Int Arch Allergy Immunol. 2013;161:197-204. 